\title{
Article
}

\section{Is Oxidative Stress an Emerging Player in the Thrombosis of Patients with Anti-Phosphatidylethanolamine Autoantibodies?}

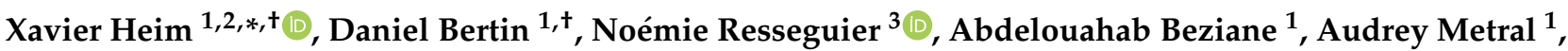 \\ Alexandre Brodovitch ${ }^{1}$, Régis Guieu ${ }^{2,4}$, Jean-Guillaume Steinberg ${ }^{2}$, Marcel Blot-Chabaud ${ }^{2}$, \\ Pierre-Emmanuel Morange ${ }^{2,5}$, Jean-Louis Mege ${ }^{1,6}$ and Nathalie Bardin ${ }^{1,2}$
}

check for updates

Citation: Heim, X.; Bertin, D.; Resseguier, N.; Beziane, A.; Metral, A.; Brodovitch, A.; Guieu, R.; Steinberg, J.-G.; Blot-Chabaud, M.; Morange, P.-E.; et al. Is Oxidative Stress an Emerging Player in the Thrombosis of Patients with Anti-Phosphatidylethanolamine Autoantibodies? J. Clin. Med. 2022, 11, 1297. https://doi.org/10.3390/ jcm11051297

Academic Editors: Muh-Shi Lin and Andrew Chih Wei Huang

Received: 30 December 2021

Accepted: 20 February 2022

Published: 27 February 2022

Publisher's Note: MDPI stays neutral with regard to jurisdictional claims in published maps and institutional affiliations.

Copyright: (C) 2022 by the authors. Licensee MDPI, Basel, Switzerland. This article is an open access article distributed under the terms and conditions of the Creative Commons Attribution (CC BY) license (https:// creativecommons.org/licenses/by/ $4.0 /)$.
1 Service d'Immunologie, Pôle de Biologie, Hôpital de la Conception, Assistance Publique-Hôpitaux de Marseille (AP-HM), 13005 Marseille, France; daniel.bertin@ap-hm.fr (D.B.);

abdelouahab.beziane@ap-hm.fr (A.B.); audrey.metral@free.fr (A.M.); alexandre.brodovitch@ap-hm.fr (A.B.); jean-louis.mege@univ-amu.fr (J.-L.M.); nathalie.bardin@univ-amu.fr (N.B.)

2 Center for CardioVascular and Nutrition Research C2VN, INSERM, INRAE Faculty of Pharmacy, Timone Campus, Aix-Marseille University, 13005 Marseille, France; regis.guieu@univ-amu.fr (R.G.); jean.steinberg@anciens.univ-amu.fr (J.-G.S.); marcel.blot-chabaud@laposte.net (M.B.-C.); pierre-emmanuel.morange@univ-amu.fr (P.-E.M.)

3 Service de Santé Publique, Unité de Recherche EA 3279, Aix-Marseille Université, 13005 Marseille, France; noemie.resseguier@univ-amu.fr

4 Service de Biochimie, Pôle de Biologie, Hôpital de la Timone, Assistance Publique-Hôpitaux de Marseille (AP-HM), 13005 Marseille, France

5 Service d'Hématologie, Pôle de Biologie, Hôpital de la Timone, Assistance Publique-Hôpitaux de Marseille (AP-HM), 13005 Marseille, France

6 Institut Hospitalo-Universitaire (IHU) Méditerranée Infection, Aix-Marseille Université, 13005 Marseille, France

* Correspondence: xavier.heim@univ-amu.fr; Tel.: +33-(0)491383907; Fax: +33-(0)491383633

+ These authors contributed equally to this work.

\begin{abstract}
The detection of anti-phosphatidylethanolamine autoantibodies (aPEs) has been proposed to improve the diagnosis and management of patients presenting clinical manifestations of antiphospholipid syndrome (APS), such as thrombosis, and who are persistently negative for conventional markers. After selecting the most specific ELISA for their detection, we evidenced the interest of aPEs in the exploration of thrombosis when APS conventional markers were negative through a 1-year retrospective study including 1131 consecutive patients routinely tested for aPEs. To validate this result, we assessed aPEs in a newly selected population of 77 patients with unexplained deep vein thrombosis (DVT). With a total prevalence of $19.5 \%$, we confirmed the interest of aPE detection in patients with unexplained DVT who were devoid of other aPLs markers. Since endosomal compartment, a source of ROS production, has been recently identified as the cellular target of aPEs in vitro, we then investigated an association between aPE positivity and reactive oxygen species (ROS) production by measuring the production of thiobarbituric acid-reactive substances. We showed, for the first time, a significant association between aPE positivity and systemic ROS production in patients which led us to hypothesize a new mechanism of action of aPEs in thrombosis through a signaling related to oxidative stress.
\end{abstract}

Keywords: reactive oxygen species (ROS); thrombosis; anti-phosphatidylethanolamine autoantibodies; ELISA; antiphospholipid syndrome; thiobarbituric acid-reactive substances (TBARs)

\section{Introduction}

The detection of autoantibodies directed against phosphatidylethanolamine (aPE) has been proposed to improve the diagnosis and management of patients presenting clinical manifestations of antiphospholipid syndrome (APS), such as thrombosis and/or obstetric diseases, and who are persistently negative for conventional markers including lupus 
coagulant (LA), IgG and/or IgM anticardiolipin autoantibodies (aCL) and IgG and/or IgM anti $\beta 2$ glycoprotein I autoantibodies (a $\beta 2 \mathrm{GPI}$ ). Although numerous antiphospholipid autoantibodies (aPL) target anionic phospholipid, aPEs are directed against a zwitterionic neutral phospholipid made up of a glycerol molecule esterified by two fatty acids and a phosphoethanolamine residue. PE is a dominant aminophospholipid of most living organisms, asymmetrically distributed in the biological membrane and mainly located in its inner leaflet [1-4]. PE is involved in cellular processes such as cell fusion, cell cycle, and autophagy [5-9]. In fact, aPEs have been proposed to be associated with a procoagulant state which support their interest as markers in the thrombotic context [10]. In a multicentric study, the prevalence of aPEs in patients with unexplained thrombosis was $15 \%$, but only $3 \%$ in controls $(p<0.0001)$ [11]. Several studies highlighted the positivity of aPEs in thrombotic events, even in the absence of conventional antiphospholipid autoantibodies (aPL), reinforcing their potential interest as diagnostic markers. Although there is a growing interest in aPEs, only a few clinical laboratories routinely test their presence. One reason for that is the lack of specificity of the commercially available ELISA kits to aPEs, as they also detect the human co-factor B2-glycoprotein I (B2GPI). Concerning the mechanism of action of these autoantibodies, it has recently been shown that early endosomes constitute their cellular target [12]. Since endosomes are of key importance for protein intracellular trafficking, it is suggested that aPEs could have a broad impact on different types of cells. These autoantibodies could especially contribute to the dysregulation of membrane redox enzymes and stimulate reactive oxygen species (ROS)-mediated signaling pathways. Oxidative stress is recognized as an important mediator of thrombotic events $[13,14]$. Therefore, it is relevant to assess ROS production in patients positive for aPEs. However, owing to its short half-life, the direct quantification of ROS is considered challenging. In contrast, ROS byproducts such as thiobarbituric acid-reactive substances (TBARs) which are formed by the peroxidation of lipids, are more stable and can be detected [15]. TBARs measure the global effects of ROS production, and more importantly the effects of tissue damage, and its level is influenced by the ratio of oxidizing/pro-oxidizing agents [16].

The aim of the present study was thus to investigate the clinical interest of aPE detection using a specific in-house-developed ELISA, and to analyze the potential association between aPE positivity and ROS production in patients with unexplained deep vein thrombosis.

\section{Materials and Methods}

\subsection{Patients}

To compare the efficiency between the in-house-developed and commercially available ELISAs for specifically detecting aPEs, we used sera from 67 patients whose samples were sent to our department at the Immunology Laboratory of Conception Hospital (AP-HM, Marseille, France) for the routine detection of IgG-aPE and IgM-aPE autoantibodies in addition to other conventional aPL. The sera were stratified as follows: 23 IgG-aPE positive (18 IgG/IgM-aB2GPI negative and $5 \mathrm{IgG}-\mathrm{aB} 2 \mathrm{GPI}$ positive), $26 \mathrm{IgG} / \mathrm{IgM}$-aPE negative (13 IgG-aB2GPI positive and 13 IgM-aB2GPI positive) and $18 \mathrm{IgM-aPE}$ positive (all IgG/IgMaB2GPI negative). The average age of patients was $50.1 \pm 18$ years, of which 18 were male and 49 were female.

A retrospective study was also performed on 2255 patients who had their aPE antibodies quantified at the Immunology Laboratory of Conception Hospital (AP-HM, Marseille, France) over a period of 1 year. Among these, 1124 patients were excluded from the study because of the lack of their clinical and biological data.

Therefore, the size of the population enrolled in the study reached 1131 patients. The collected clinical and biological data were the following: age, sex, medical departments, dosage of IgG- and IgM-anti-B2GPI, IgG- and IgM-aCL, platelet count, number of thromboses and their characteristics (recurrence, type of thrombosis), and obstetric complications (fetal loss, postpartum hemorrhage, pre-eclampsia, and infertility). 
Arterial thromboses included: myocardial infarction, stroke, or any other acute thrombotic event involving the arterial vascular system.

Venous thromboses included: deep vein thrombosis, pulmonary embolism, or any other acute thrombotic event involving the venous vascular system.

An event was considered as recurrent when the number of events was greater than or equal to 2 .

To confirm the interest of quantifying IgG- and IgM-aPE antibodies in patients with clinical symptoms of APS in the absence of conventional autoantibodies, a cohort of 77 patients with deep venous thrombosis (DVT) who were negative for conventional antiphospholipid autoantibodies were selected after careful clinical examination and normal biological screening including prothrombin time (PT), activated partial thromboplastin time (aPTT), D-dimer, fibrinogen, factor VIII level, lupus anticoagulant (LA), anti-cardiolipin, and anti- $\beta 2$ glycoprotein I antibodies. LA was assessed using two clotting times: partial thromboplastin time-lupus anticoagulant (PTT-LA) and diluted Russel viper venom time (dRVVT). Inherited thrombophilia etiologies were also ruled out by researching antithrombin (AT), protein C (PC) and protein S (PS) deficiencies, factor V Leiden (FVL), and the G20210A prothrombin mutation (PTM).

All samples were taken from a declared Biobank (DC 2012-1704) in compliance with ethical directives. This study was approved and registered by the Assistance Publique Hôpitaux de Marseille and fulfilled local requirements in terms of data collection, patients ${ }^{\prime}$ consent, and protection of data (RGPD/APHM 2019-108). This study was conducted according to the Declaration of Helsinki.

\section{2. aPE Detection}

\subsubsection{In-House ELISA with PE-Only Coating}

Irradiated polystyrene microtiter plates (Maxisorp ${ }^{\circledR}, \mathrm{Nunc}^{\circledR}$, Rockilde, Denmark) were coated $(30 \mu \mathrm{L} /$ well) either with a solution containing $50 \mu \mathrm{g} / \mathrm{mL}$ PE from egg yolk (SigmaAldrich, Saint-Quentin-Fallavier, France) or with the solvent absolute ethanol ( $>99.7 \%$, VWR Chemicals Prolabo), used to determine the non-specific binding of each sample. The plates were dried by evaporation at $18{ }^{\circ} \mathrm{C}$ and blocked by incubating with $10 \%$ fetal calf serum (FCS, GIBCO ${ }^{\circledR}$, Invitrogen, Cergy-Pontoise, France) in PBS for $1 \mathrm{~h}$ at room temperature (RT). Finally, plates were dried using nitrogen gas.

Sera were diluted 1:100 in 10\% FCS-PBS and added in duplicate to coated and noncoated wells (100 $\mu \mathrm{L} /$ well) and incubated for $1 \mathrm{~h}$ at RT. After the washing step (three times with PBS), $100 \mu \mathrm{L}$ of alkaline-phosphatase-conjugated goat anti-human immunoglobulin (Jackson ImmunoResearch Laboratories, West Grove, PA, USA) IgG (diluted 1:5000 in PBSFCS) or IgM (diluted 1:2400 in PBS-FCS) were added in each well. Following $1 \mathrm{~h}$ incubation at RT and the washing step, a phosphatase substrate para-nitrophenylphosphate, $\mathrm{pNPP}$ (Sigma-Aldrich, Saint-Quentin-Fallavier, France) $(1 \mathrm{mg} / \mathrm{mL}$ in $1 \mathrm{M}$ diethanolamine buffer, $\left.0.5 \mathrm{mM} \mathrm{MgCl}_{2}, \mathrm{pH}=9.8\right)$ was added $(100 \mu \mathrm{L} /$ well $)$ and the plate was incubated at $37^{\circ} \mathrm{C}$ for $15 \mathrm{~min}$ (IgG-aPE) or $20 \mathrm{~min}$ (IgM-aPE). Then, optical density (OD) was measured at $405 \mathrm{~nm}$. OD for each sample was obtained by subtracting the OD of the non-coated well from that with antigen. The cut-off levels for IgG-aPE and IgM-aPE were determined with 100 sera from age- and sex-matched healthy blood donors, corresponding to the 99th percentile of control group values. These cut-off values were 0.47 and $0.68 \mathrm{OD}$, corresponding to the following arbitrary units: 18 and $59 \mathrm{U} / \mathrm{mL}$ for IgG-aPE and IgM-aPE, respectively.

\subsubsection{Commercial aPE ELISA with PE and B2GPI Coating}

We used the commercial kit provided by Theradiag (Marne-la-Vallée, France) and manufactured by Aesku (Wendelsheim, Germany). As mentioned by the manufacturer and highlighted in the datasheet, AESKULISA Ethanolamine-GM is a solid-phase enzyme immunoassay coated with purified phosphatidylethanolamine and native human B2GPI proteins. 


\subsection{Adsorption Experiments on B2GPI Coated Plate}

The adsorption experiments were carried out as follows. Briefly, sera were selected for IgG-aB2GPI positivity as confirmed by the IgG-aB2GPI ELISA kit from Orgentec. Two hundred microliters of diluted patient's sera (1:100) were added to $1 \mu \mathrm{g}$ B2GPI-coated plate (human $\beta_{2}$-glycoprotein, the binding site) and incubated for $1 \mathrm{~h}$ at RT. After the first adsorption, a second adsorption was carried out by the same procedure and this adsorption procedure was repeated identically up to four times. The efficiency of the adsorption procedure was verified by the IgG-aB2GPI assay on the sera processed before and after the adsorption experiments.

\subsection{TBARs Dosage}

Serum TBARs concentration was assessed according to Uchiyama and Mihara method [15] modified by Jammes [17]. After the centrifugation of blood samples $\left(1500 \times g\right.$ at $4{ }^{\circ} \mathrm{C}$ for $10 \mathrm{~min}$ ), $300 \mu \mathrm{L}$ of serum was deproteinized in 10\% trichloroacetic acid (TCA, v/v) containing $2.90 \mathrm{mmol}$ of ethanolicbutylatedhydroxytoluene (Sigma-Aldrich, Saint-Quentin Fallavier, France) to avoid further peroxidation. After vortexing and centrifugation $\left(2500 \times g\right.$ at $4{ }^{\circ} \mathrm{C}$ for $\left.15 \mathrm{~min}\right)$, the supernatants were stored at $-80{ }^{\circ} \mathrm{C}$ until use. Samples were then aliquoted $(200 \mu \mathrm{L})$ and $200 \mu \mathrm{L}$ of $8.1 \%$ sodium dodecylsulfate, $1.5 \mathrm{~mL}$ of $20 \%$ acetate buffer $(\mathrm{pH}=3.5), 1.5 \mathrm{~mL}$ of freshly prepared $0.8 \%$ thiobarbituric acid (SigmaAldrich Co.) and $600 \mu \mathrm{L}$ of water were then successively added. The test tubes containing glass beads were heated at $100{ }^{\circ} \mathrm{C}$ for $60 \mathrm{~min}$ and cooled with tap water. Then, $4 \mathrm{~mL}$ of $\mathrm{n}$-butanol and $1 \mathrm{~mL}$ of water were added into each tube. After vortexing for $5 \mathrm{~min}$, the mixture was centrifugated $(2000 \times g$ for $3 \mathrm{~min})$ to obtain a rapid separation between organic and aqueous phases. The upper organic phase was pipetted off and the pink pigment was measured using a spectrofluorimeter at an excitation wavelength of $515 \mathrm{~nm}$ and an emission wavelength of $553 \mathrm{~nm}$ (Shimadzu model RF5000; Shimadzu, Kyoto, Japan). A standard curve of TBARs was obtained after overnight hydrolysis in a solution containing $1 \mathrm{mmol}$ of tetraethoxypropane (Sigma-Aldrich Co.) in $0.1 \mathrm{~N} \mathrm{HCl}$ at RT.

\subsection{Statistical Analysis}

Statistical analysis comparing the positivity or negativity of IgG and IgM aPE with the presence or absence of the other studied parameters was performed using the Chi-2 method. The concordance between the in-house-developed and commercial aPE ELISA was evaluated using Cohen's Kappa coefficient, which takes on the value: zero if there is no more agreement between two tests than expected by chance; 1 if there was a perfect agreement. Kappa values below 0.4 were considered as poor agreement. Paired sample $t$-test was used to compare aPE ELISA optical densities before and after serum adsorption on B2GPI. Student's $t$-test was used to compare the mean values of TBARs levels. Dot plots, box plots and statistical calculations were performed with GraphPad Prism version 5.03 (GraphPad Software, La Jolla, CA, USA). The threshold for statistical significance was set at $p=0.05$. Effect size (Cohen's $\mathrm{d}$ ) and power value were calculated using pwr library of $\mathrm{R}$ software version 3.6.0 ( $\mathrm{R}$ Foundation for Statistical Computing).

\section{Results}

\subsection{Specific Detection of aPE}

To check ELISA specificity for aPE detection, we first compared our in-house ELISA to the commercial one. To this end, sera from 67 patients explored in the routine practice were analyzed. From the 23 patients who tested positive for IgG-aPE in the in-house ELISA, only 8 sera were positive when tested using the commercial kit. Besides, among the $18 \mathrm{IgM}$-aPE positive sera, only 5 were found to be positive using the commercial kit (Figure 1). Accordingly, the kappa coefficient was 0.042, showing poor concordance between the two methods. Since human B2GPI was coated with PE in the commercial ELISA, adsorption experiments were conducted on immobilized B2GPI to further explore discrepancies between the two ELISA systems (Figure 2). Five positive sera with the in- 
house ELISA (three positive and two negative with the commercial ELISA) were tested with aPE ELISA before and after the adsorption test. All of them were aB2GPI positive. After the adsorption procedure, their reactivity towards PE significantly decreased in the commercial ELISA but not with the in-house aPE ELISA. This shows that the reactivity obtained with commercial kit could be partly related to a reactivity against the B2GPI, excluding its use for specific aPE detection.

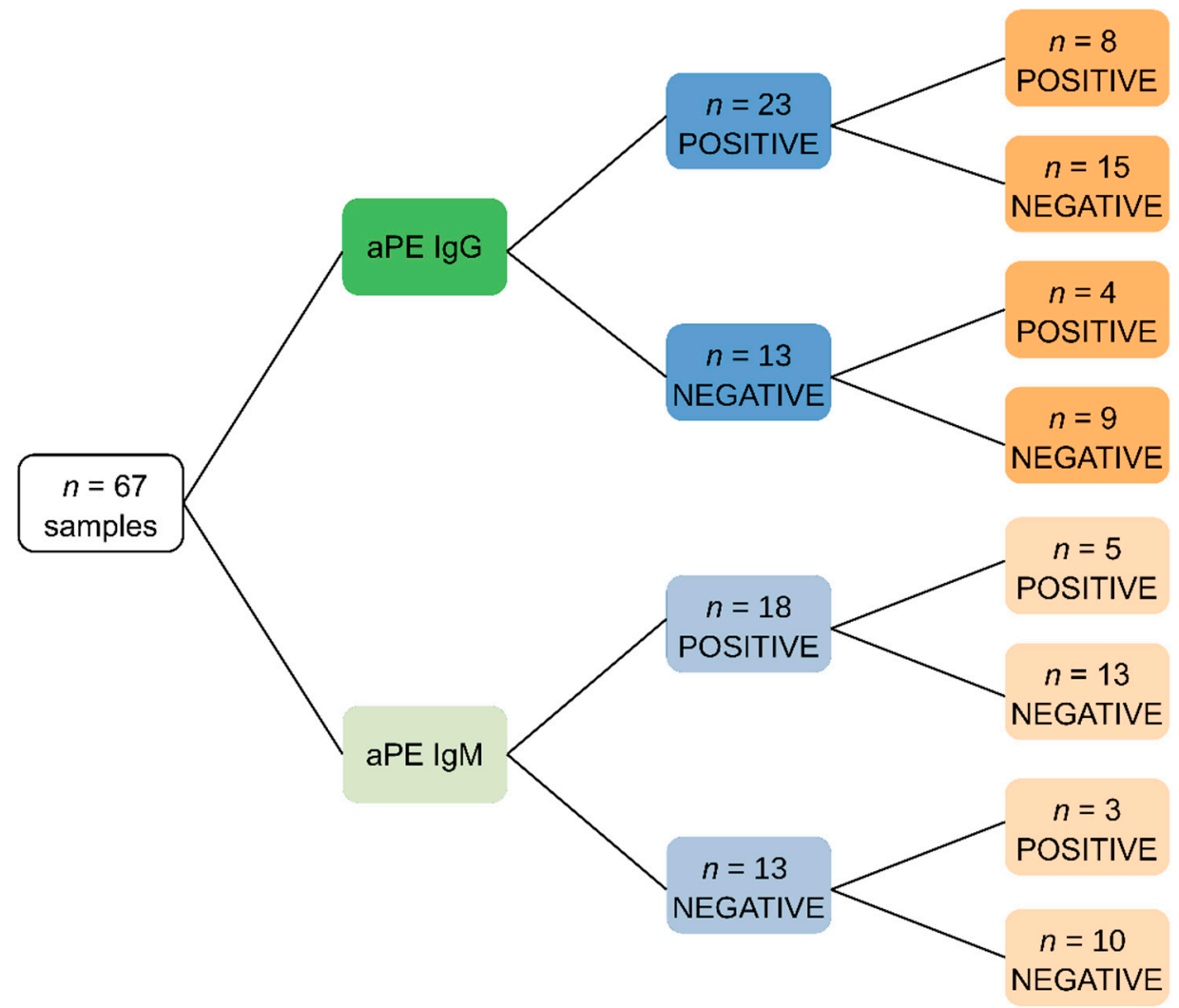

Figure 1. Tree representation of results obtained using different ELISA assays. Tree diagram showing serological status of patients towards phosphatidylethanolamine according to in-house or commercial aPE ELISA. aPEs: anti-phosphatidylethanolamine autoantibodies.

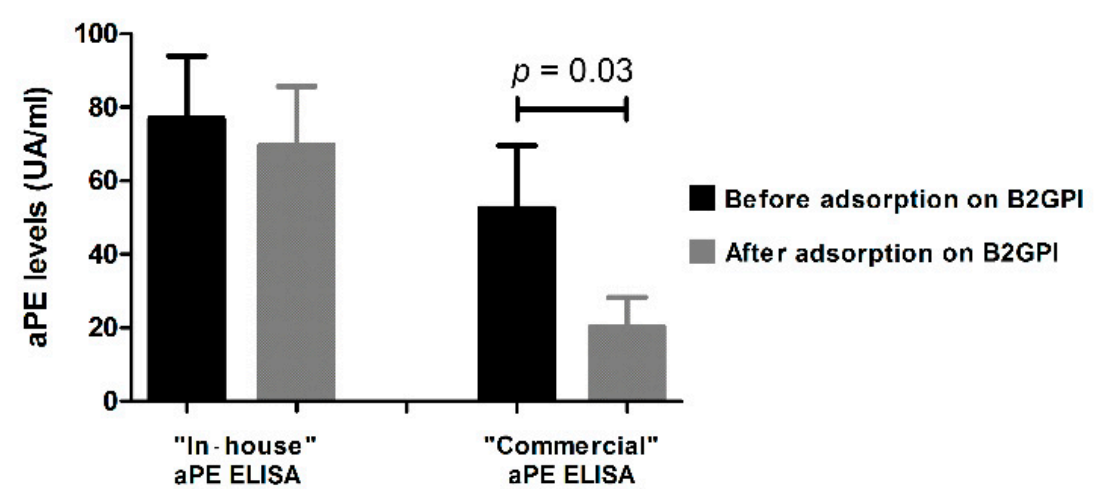

Figure 2. Comparison of measured optical densities (OD) between in-house and commercial aPE ELISA. Comparison of measured optical densities (OD) between in-house and commercial IgG aPE ELISA. Measurements were performed before and after adsorption of sera on immobilized beta-2-glycoprotein I (B2GPI). Adsorption did not significantly decrease the measured OD with the in-house ELISA, whereas OD was significantly decreased with the commercial aPE ELISA ( $p=0.03$, paired samples $t$-test). Bars represents means and whiskers represents SEM. aPE: antiphosphatidylethanolamine autoantibodies. 


\subsection{Retrospective Clinical Study}

Sera from a total of 1131 patients including 848 women (75\%) and 283 men (25\%), with a mean age of $46 \pm 16$ years were used to quantify aPE antibodies using the inhouse-developed ELISA. Patients' serum samples were addressed to our laboratory by departments of neurology $(35.2 \%)$, internal medicine $(32.6 \%)$, thrombosis exploration $(15.1 \%)$, dermatology $(8.8 \%)$, hematology (2.7), infectious diseases $(2.4 \%)$, gynecology $(1.2 \%)$, nephrology $(1.1 \%)$, post-emergency medicine $(1.1 \%)$ and others (intensive care, gastro-enterology, cardiology, rheumatology, surgery, otorhinolaryngology, addictology, odontology, dermatology, psychiatry, oncology). Geologically, all patients were from southeast France.

Clinical and biological data collected from all patients (Table 1 ) revealed that $25.7 \%$ of patients had at least one thrombosis, $21.0 \%$ of women had at least two fetal losses, $2.9 \%$ infertility, and $3.5 \%$ had other obstetrical complications. All conventional aPL markers were assessed and were of low prevalence. In contrast, the prevalence in patients with aPE, (IgG and/or IgM) was 20.7\% (234/1131), of which 89\% had IgG-aPE (209/234) and $16 \%$ had IgM-aPE (38/234). Among them, 99 patients positive for aPEs had an additional dosage at least 12 weeks after they were observed. This allowed us to measure antibody persistence rate and we show it to be $67 \%(33 / 99)$.

Table 1. Clinical and biological data of the population tested for aPEs.

\begin{tabular}{|c|c|c|c|}
\hline $\begin{array}{l}\text { Clinical or } \\
\text { Biological } \\
\text { Parameters }\end{array}$ & $\begin{array}{l}\text { Number of Positive } \\
\text { Patient for Each } \\
\text { Parameter }\end{array}$ & $\begin{array}{l}\text { Size of Population } \\
\text { (Nb of Patients) }\end{array}$ & Prevalence \\
\hline \multicolumn{4}{|l|}{ Clinical events } \\
\hline -Thrombosis: & 291 & 1131 & 0.257 \\
\hline -Arterial thrombosis & 143 & 291 & 0.491 \\
\hline -Venous thrombosis & 181 & 291 & 0.622 \\
\hline -Cerebral stroke & 92 & 291 & 0.316 \\
\hline $\begin{array}{l}\text {-Pulmonary } \\
\text { embolism }\end{array}$ & 76 & 291 & 0.261 \\
\hline $\begin{array}{l}\text {-Myocardial } \\
\text { infarction }\end{array}$ & 12 & 291 & 0.041 \\
\hline -Recurrent fetal losses & 178 & 848 & 0.21 \\
\hline $\begin{array}{l}\text {-Obstetrical } \\
\text { complications }\end{array}$ & 30 & 848 & 0.035 \\
\hline -Infertility & 25 & 848 & 0.029 \\
\hline \multicolumn{4}{|l|}{ Biological data } \\
\hline aPE IgG and/or IgM & 234 & 1131 & 0.207 \\
\hline aPE IgG & 209 & 1131 & 0.185 \\
\hline aPE IgM & 38 & 1131 & 0.034 \\
\hline aB2GPI IgG & 44 & 1131 & 0.039 \\
\hline aB2GPI IgM & 41 & 1131 & 0.036 \\
\hline aCL IgG & 60 & 1131 & 0.053 \\
\hline $\mathrm{aCL} \operatorname{IgM}$ & 84 & 1131 & 0.074 \\
\hline LA & 66 & 968 & 0.068 \\
\hline
\end{tabular}

As presented in Table 2, an association between aPEs and thrombosis was near statistical significance $(p=0.06)$, but no clear association with fetal loss $(p=0.83)$, or other obstetrical complications $(p=0.12)$ were obtained. We also noted a trend towards statistical 
significance between the association of aPEs and infertility $(p=0.06)$. Clinical association was further analyzed according to the aPE isotype. No significant association was found between IgM-aPE and all the clinical contexts described in Table 2. In contrast, we found a significant association between IgG-aPE prevalence and thrombosis $(p=0.04)$ but no association with obstetrical manifestations (Table 2). No preferential association between IgG aPE and type of thrombosis (arterial or venous, cerebral stroke, pulmonary embolism, myocardial infarction) was found.

Table 2. Clinical events according to aPE serological profile.

\begin{tabular}{|c|c|c|c|c|c|c|c|c|c|}
\hline & \multicolumn{3}{|c|}{ aPE IgG and/or IgM } & \multicolumn{3}{|c|}{ aPE IgG } & \multicolumn{3}{|c|}{ aPE IgM } \\
\hline & $\mathrm{Nb}(\%)$ & $\mathrm{Nb} \stackrel{+}{(\%)}$ & $p$ value & $\mathrm{Nb}(\%)$ & $\mathrm{Nb} \stackrel{+}{(\%)}$ & $p$ value & $\mathrm{Nb}(\%)$ & $\mathrm{Nb}_{(\%)}^{+}$ & $p$ value \\
\hline Thrombosis & $220(24.5 \%)$ & $71(30.3 \%)$ & 0.06 & $226(24.5 \%)$ & $65(31.1 \%)$ & 0.04 & $281(25.7 \%)$ & $10(26.3 \%)$ & 0.93 \\
\hline $\begin{array}{c}\text { Recurrent } \\
\text { pregnancy } \\
\text { loss }\end{array}$ & $139(20.8 \%)$ & $39(21.6 \%)$ & 0.83 & $146(21.2 \%)$ & $32(20.1 \%)$ & 0.76 & $169(20.7 \%)$ & $9(28.1 \%)$ & 0.31 \\
\hline $\begin{array}{l}\text { Obstetrical } \\
\text { complica- } \\
\text { tions }\end{array}$ & $27(4.1 \%)$ & $3(1.7 \%)$ & 0.12 & $27(3.9 \%)$ & $3(1.9 \%)$ & 0.21 & $30(3.7 \%)$ & $0(0 \%)$ & 0.62 \\
\hline Infertility & $16(2.4 \%)$ & $9(5.0 \%)$ & 0.06 & $17(2.5 \%)$ & $8(5.0 \%)$ & 0.11 & $23(2.8 \%)$ & $2(6.3 \%)$ & 0.24 \\
\hline
\end{tabular}

aPEs: anti-phosphatidylethanolamine autoantibodies, $\mathrm{Nb}$ : number of patients.

Among these IgG aPE-positive patients with a history of thrombosis, 34\% (22/65) were positive for conventional aPL, and the majority of them $(66 \%, 43 / 65)$ had unexplained thrombosis without conventional aPL markers.

In this subgroup of patients without conventional aPL, we found 24 patients with deep vein thrombosis, 12 strokes, and 9 with pulmonary embolism. A total of 26 patients had isolated thrombosis and 17 patients had recurrent thrombosis.

Interestingly, we found a significant correlation between the level of IgG-aPE and the presence of thrombosis $(p=0.03)$. The higher the level of IgG-aPE, the greater the risk of developing thrombosis. Moreover, the level of IgG-aPE was significantly associated with the recurrence of thrombotic events $(p=0.02)$. (Figure 3).

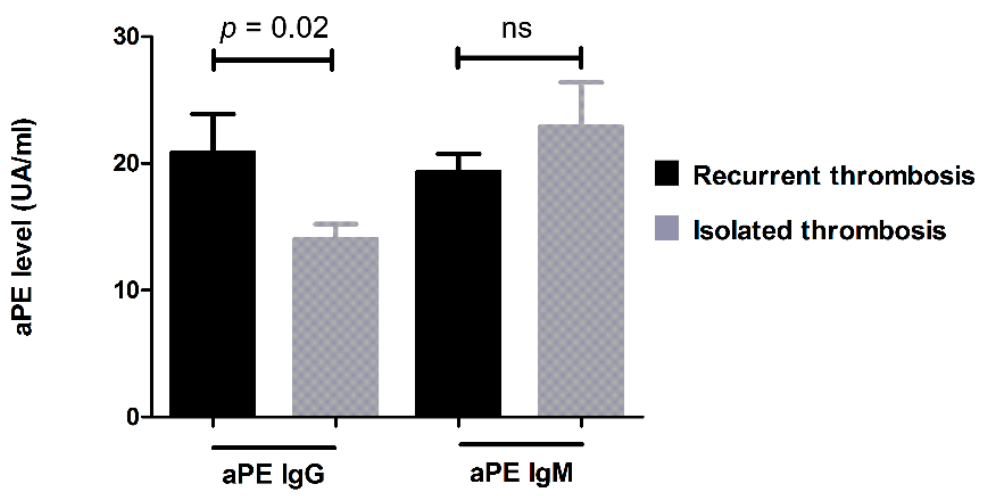

Figure 3. Quantitative study of aPEs in thrombotic patients. Comparison of aPE levels for each isotype in patients suffering from isolated or recurrent thrombosis. aPEs: anti-phosphatidylethanolamine autoantibodies; ns: not significant. Bars represents means and whiskers represents SEM.

\subsection{Association between aPEs and Oxidative Stress in Patients with Unexplained DVT}

As it has been reported that aPEs could stimulate the ROS pathway, we investigated whether the production of ROS was associated with aPE positivity. As unexplained thrombosis is one of the most prevalent clinical contexts associated with aPE positivity, we explored 77 patients with unexplained DVT, all negative for conventional aPL. We found a total prevalence of $19.5 \%$ for aPEs (14.3\% IgG-aPE and 5.2\% IgM-aPE) which confirms the results obtained from the retrospective study. We then measured the concentration of TBARs in the serum of patients with DVT. According to the availability of the samples, 
a total of 22 samples were tested. In these experiments, the concentration of TBARs informed the systemic level of lipoperoxydation resulting from ROS production. As shown in Figure 4, the concentration of TBARs was significantly higher in thrombotic aPE-positive patients than in those negative for aPEs (effect size and power value were 1.04 and 0.637, respectively).

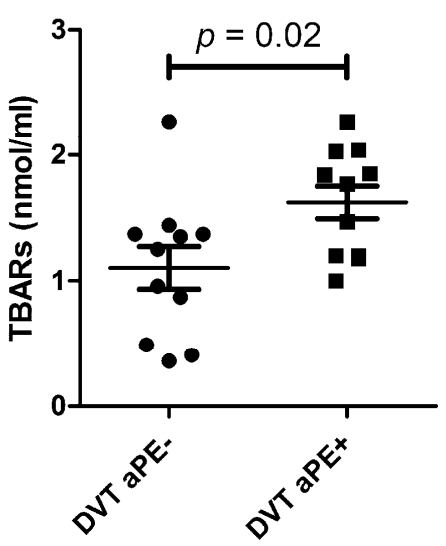

Figure 4. Comparison of TBARs levels in sera of patients with deep vein thrombosis. Comparison of TBARs levels in sera of patients with deep vein thrombosis (DVT) according to their aPE serological status. The largest horizontal line shows the mean value, and whiskers represent SEM. Serum levels of TBARs were significantly higher in aPE+ DVT patients than aPE- DVT patients $(p=0.02$, unpaired $t$-test). TBARs: thiobarbituric acid-reactive substances; aPEs: anti-phosphatidylethanolamine autoantibodies; DVT: deep vein thrombosis.

\section{Discussion}

The interest of using aPEs as biomarkers has been poorly studied in the literature and actions for their detection are not systematically performed in routine clinical practice. In this study, we evidenced the interest of aPEs in the exploration of APS, especially when conventional markers were negative, using the results obtained from 1131 unselected patients and 77 selected patients with unexplained DVT. We showed, for the first time in patients with unexplained thrombosis, that oxidative stress was higher in aPE-positive than in aPE-negative patients, raising an interesting hypothesis on the mechanism of action of aPE in thrombosis.

Other studies have highlighted the importance of testing aPEs in cases of thrombosis $[10,11]$. In particular, results concerning prevalence and isolated positivity of aPEs (without conventional aPL) are in accordance with our findings. For instance, Sanmarco et al. [10] found a significantly higher prevalence of aPE (18\%) in patients with unexplained thrombosis. In the same way, Sanmarco et al. showed that aPEs were the aPL with the highest odd ratio for thrombosis ([OR]: 4.2; $p=0.001$ ) [11]. In our study, we additionally found a significant association between the level of $\operatorname{IgG}$ aPE and the recurrence of the thrombotic event. Although studies had previously shown an association between aPEs and obstetric complications [18], such an association was not observed in our study performed on a routine population of unselected patients. In addition, we found an association with a trend towards statistical significance between aPEs and infertility. This result is in agreement with a prospective study that studied the prevalence of aPL in 101 women who underwent in vitro fertilization, and showed that aPEs were the most prevalent aPL [19].

Until now, PE has appeared as a major anticoagulant factor involved in the anticoagulant $\mathrm{C}$ protein pathway. In fact, it has been suggested that the inhibitory effect of aPEs on this anticoagulant pathway is one of the mechanisms leading to thrombosis [20-22]. However, the patients enrolled in our study had normal protein $C$ activity. This apparent discrepancy may be explained by the fact that PC activity is an in vitro chronometric assay performed in the presence of an excess of phospholipids, which prevents aPEs from interfering with PC activity measurement. 
Here, we showed for the first time that aPE-mediated thrombotic effects are regulated through ROS signaling. Indeed, we found that TBARs production was significantly higher in aPE-positive thrombotic patients than in negative ones. Intracellular ROS such as superoxide anion and hydrogen peroxide are directly involved in platelet activation and thrombus formation $[13,14]$. Thus, the overproduction of ROS may be associated with diseases such as hypercholesterolemia, diabetes, hypertension and metabolic syndrome, all risk factors for thrombosis. Hou et al. demonstrated that PE antigen may be accessible in early endosomes, by using different PE-binding agents including plasma from an aPEpositive patient and purified aPEs [12]. The authors suggested that these autoantibodies could act through ROS generation. This agrees with our results obtained from thrombotic patients, showing that aPEs are involved in ROS production, which themselves contribute to thromboses. In this study, we also emphasized the relevance of using highly specific aPEdetection ELISA kits. Indeed, our experiments show that with the commercially available ELISA assays, a great number of positive patients can be attributed to the presence of confounding antibodies directed against B2GPI. The development of commercial ELISA kits specific for PE is thus required.

In conclusion, we demonstrated that aPEs should be assessed in thrombotic patients, especially when other aPLs markers are absent. The detection must be performed by using an ELISA with PE as the sole coating antigen. Finally, we show for the first time an association between aPE positivity and the systemic effect of ROS generation, suggesting a new mechanism of action of aPEs through ROS signaling in the thrombotic process. Studies are now necessary to confirm this concept in a larger cohort of patients and pave the way for investigating novel therapeutic strategies.

Author Contributions: N.B., D.B., X.H., R.G., P.-E.M. contributed to the conception and design of the study; D.B., A.M., X.H., J.-G.S. and A.B. (Abdelouahab Beziane) performed the data collection; N.R., D.B., N.B., A.B. (Alexandre Brodovitch) and X.H. performed the statistical analysis; N.B., D.B., J.-L.M., M.B.-C. and X.H. wrote the first draft of the manuscript; N.B., X.H., D.B., N.R. and J.-G.S. wrote sections of the manuscript. All authors have read and agreed to the published version of the manuscript.

Funding: This research did not receive any specific grant from funding agencies in the public, commercial, or non-profit sectors.

Institutional Review Board Statement: All samples were part from a declared Biobank (DC 20121704) in compliance with ethical directives. This study was approved and registered by the Assistance Publique Hôpitaux de Marseille and fulfilled local requirements in terms of data collection, patients' consent, and protection of data (RGPD/APHM 2019-108). This study was conducted according to the Declaration of Helsinki.

Informed Consent Statement: Not applicable.

Data Availability Statement: The data used to support the findings of this study are available from the corresponding author upon request.

Acknowledgments: We thank Théradiag (Marne la Vallée, France) and KeyOfLab (formerly known as Orgentec SASU ${ }^{\circledR}$, Trappes, France) for providing ELISA kits for anti-phosphatidylethanolamine and anti-beta-2 glycoprotein I autoantibodies detection. We also thanks Ahmad Joshkon for proofreading and editing this manuscript.

Conflicts of Interest: The authors declare no conflict of interest.

\section{References}

1. Langley, K.E.; Kennedy, E.P. Energetics of rapid transmembrane movement and of compositional asymmetry of phosphatidylethanolamine in membranes of Bacillus megaterium. Proc. Natl. Acad. Sci. USA 1979, 76, 6245-6249. [CrossRef]

2. Li, Z.; Wells, C.W.; North, P.E.; Kumar, S.; Duris, C.B.; McIntyre, J.A.; Zhao, M. Phosphatidylethanolamine at the Luminal Endothelial Surface-Implications for Hemostasis and Thrombotic Autoimmunity. Clin. Appl. Thromb. Hemost 2011, 17, 158-163. [CrossRef] 
3. Zachowski, A. Phospholipids in animal eukaryotic membranes: Transverse asymmetry and movement. Biochem. J. 1993, 294 Pt 1, 1-14. [CrossRef] [PubMed]

4. Zwaal, R.F.; Roelofsen, B.; Comfurius, P.; van Deenen, L. Organization of phospholipids in human red cell membranes as detected by the action of various purified phospholipases. Biochim. Biophys. Acta 1975, 406, 83-96. [CrossRef]

5. Deeba, F.; Tahseen, H.N.; Sharad, K.S.; Ahmad, N.; Akhtar, S.; Saleemuddin, M.; Mohammad, O. Phospholipid diversity: Correlation with membrane-membrane fusion events. Biochim. Biophys. Acta 2005, 1669, 170-181. [CrossRef]

6. Verkleij, A.J.; Leunissen-Bijvelt, J.; de Kruijff, B.; Hope, M.; Cullis, P.R. Non-Bilayer Structures in Membrane Fusion. Ciba Found. Symp. 1984, 103, 45-59. [CrossRef] [PubMed]

7. Emoto, K.; Kobayashi, T.; Yamaji, A.; Aizawa, H.; Yahara, I.; Inoue, K.; Umeda, M. Redistribution of phosphatidylethanolamine at the cleavage furrow of dividing cells during cytokinesis. Proc. Natl. Acad. Sci. USA 1996, 93, 12867-12872. [CrossRef]

8. Hailey, D.W.; Rambold, A.S.; Satpute-Krishnan, P.; Mitra, K.; Sougrat, R.; Kim, P.K.; Lippincott-Schwartz, J. Mitochondria Supply Membranes for Autophagosome Biogenesis during Starvation. Cell 2010, 141, 656-667. [CrossRef]

9. Nebauer, R.; Rosenberger, S.; Daum, G. Phosphatidylethanolamine, a Limiting Factor of Autophagy in Yeast Strains Bearing a Defect in the Carboxypeptidase Y Pathway of Vacuolar Targeting. J. Biol. Chem. 2007, 282, 16736-16743. [CrossRef]

10. Sanmarco, M.; Alessi, M.-C.; Harle, J.R.; Sapin, C.; Aillaud, M.-F.; Gentile, S.; Juhan-Vague, I.; Weiller, P.-J. Antibodies to phosphatidylethanolamine as the only antiphospholipid antibodies found in patients with unexplained thromboses. Thromb. Haemost. 2001, 85, 800-805. [CrossRef]

11. Sanmarco, M.; Gayet, S.; Alessi, M.-C.; Audrain, M.; de Maistre, E.; Gris, J.-C.; de Groot, P.G.; Hachulla, E.; Harlé, J.-R.; Sié, P.; et al. Antiphosphatidylethanolamine antibodies are associated with an increased odds ratio for thrombosis. A Multicenter Study with the Participation of the European Forum on Antiphospholipid Antibodies. Thromb. Haemost. 2007, 97, 949-954. [CrossRef] [PubMed]

12. Hou, S.; Fölsch, H.; Ke, K.; Mills, J.C.; Ramsey-Goldman, R.; Zhao, M. Early endosome as a pathogenic target for antiphosphatidylethanolamine antibodies. Proc. Natl. Acad. Sci. USA 2017, 114, 13798-13803. [CrossRef] [PubMed]

13. Qiao, J.; Arthur, J.F.; Gardiner, E.E.; Andrews, R.K.; Zeng, L.; Xu, K. Regulation of platelet activation and thrombus formation by reactive oxygen species. Redox Biol. 2018, 14, 126-130. [CrossRef]

14. Gutmann, C.; Siow, R.; Gwozdz, A.M.; Saha, P.; Smith, A. Reactive Oxygen Species in Venous Thrombosis. Int. J. Mol. Sci. 2020, 21, 1918. [CrossRef]

15. Mihara, M.; Uchiyama, M. Effects of antioxidants on the TBA reaction of various rat liver homogenates. Biochem. Med. 1983, 30, 131-134. [CrossRef]

16. Yoshikawa, T.; Tanaka, H.; Kondo, M. The increase of lipid peroxidation in rat adjuvant arthritis and its inhibition by superoxide dismutase. Biochem. Med. 1985, 33, 320-326. [CrossRef]

17. Jammes, Y.; Steinberg, J.G.; Mambrini, O.; Brégeon, F.; Delliaux, S. Chronic fatigue syndrome: Assessment of increased oxidative stress and altered muscle excitability in response to incremental exercise. J. Intern. Med. 2005, 257, 299-310. [CrossRef]

18. Gris, J.C.; Quéré, I.; Sanmarco, M.; Boutiere, B.; Mercier, E.; Amiral, J.; Hubert, A.M.; Ripart-Neveu, S.; Hoffet, M.; Tailland, M.L.; et al. Antiphospholipid and antiprotein syndromes in non-thrombotic, non-autoimmune women with unexplained recurrent primary early foetal loss. The Nîmes Obstetricians and Haematologists Study-NOHA. Thromb. Haemost. 2000, 84, 228-236. [PubMed]

19. Sanmarco, M.; Bardin, N.; Camoin, L.; Beziane, A.; Gamerre, M.; Porcu, G.; Dignat-George, F. Antigenic Profile, Prevalence, and Clinical Significance of Antiphospholipid Antibodies in Women Referred for in Vitro Fertilization. Ann. N. Y. Acad. Sci. 2007, 1108, 457-465. [CrossRef] [PubMed]

20. Esmon, N.L.; Safa, O.; Smirnov, M.D.; Esmon, C.T. Antiphospholipid Antibodies and the Protein C Pathway. J. Autoimmun. 2000, 15, 221-225. [CrossRef] [PubMed]

21. Smirnov, M.D.; Safa, O.; Regan, L.; Mather, T.; Stearns-Kurosawa, D.J.; Kurosawa, S.; Rezaie, A.R.; Esmon, N.L.; Esmon, C.T. A Chimeric Protein C Containing the Prothrombin Gla Domain Exhibits Increased Anticoagulant Activity and Altered Phospholipid Specificity. J. Biol. Chem. 1998, 273, 9031-9040. [CrossRef] [PubMed]

22. Smirnov, M.D.; Esmon, C.T. Phosphatidylethanolamine incorporation into vesicles selectively enhances factor Va inactivation by activated protein C. J. Biol. Chem. 1994, 269, 816-819. [CrossRef] 